\title{
Zodiacal Light - Scattered Fraunhofer Line Profiles
}

\section{David Clarke}

University Observatory, Acre Rd., GLASGOW G20 OTL, Scotland, U.K.

\begin{abstract}
The scattering function of Hong's successful model for the brightness variation with elongation, $B(\epsilon)$, of the Zodiacal Light has been used to calculate profiles of Doppler shifted Fraunhofer lines. Substantial line shape changes with $\epsilon$ are predicted with an indication of a bifurcation at $\epsilon<30^{\circ}$, so offering an explanation for reported $F$ corona observations.
\end{abstract}

\section{Introduction}

The interplanetary particles giving rise to the Zodiacal Light (ZL) are in motion and, as a consequence, the scattered solar radiation suffers Doppler effects. The original solar Fraunhofer lines are displaced from their rest positions and are distorted. Records of the ZL spectra have been obtained using conventional diffraction grating spectrometers [e.g. see Blackwell \& Ingham, 1961], scanning Fabry+Pérot interferometers [e.g. see Ring et al., 1964] and a correlation mask radial velocity spectrometer (CMRVS) [see Fried, 1978].

The bterature reveals contention over interpretations of the various observations. There are differences between results according to the experimental technique and the conclusions on radial velocities (RVs) are diverse. For example, Fried (1978) found evening/morning symmetry with opposite senses and with motions in excess of those for circular or elliptical Keplerian orbits. For particles within 0.7AU from the Sun, the orbital speeds were claimed to be above the Solar System escape velocity. To the contrary, East \& Reay (1984) reported asymmetry between the evening/morning elongations and were critical of Fried's latter conclusion.

In this paper it is demonstrated that the phase angle scattering function associated with the interplanetary particles has strong influence on the way the chosen observational technique affects the basic recorded velocities and on how data are interpreted in terms of orbital velocities.

\section{Doppler Effects and Hong's Scattering Function}

According to James' (1969) bench mark model, Doppler affected ZL line profiles are fairly insensitive to the particle scattering function. Only simple functions have been explored, however, and without reference to models which describe the behaviour of the brightness, $B(\epsilon)$, and polarization, $p(\epsilon)$ with elongation. Hong (1985) has slown that $B(\epsilon)$ can be modelled by invoking a dust mixture of three kinds of particle with phase angle characteristics described by Heyney- 
Greenstein functions. It was therefore decided to explore the effects of the Hong mixture on the prediction for ZL line profiles using the natural extension of James' model. [A more detailed account of the study is found in Clarke, et al. (1995)].

By using a local particle number density given by $n(r) \propto r^{-\beta}$ with a grain size distribution $f(s)$, independent of solar distance, the scattered radiation from a volume may be described in terms of a cross-section distribution, $\sigma(s)$ with associated phase functions, $\phi(\Theta, s)$. Integrating along the line of sight leads to:

$$
B_{\lambda}(\epsilon)=\frac{F_{0} r_{0} n_{0} \bar{\sigma}}{\sin ^{\beta+1}(\epsilon)} \int_{\epsilon}^{\pi} Z(\lambda) \Phi(\Theta) \sin ^{\beta}(\Theta) d \Theta
$$

where $F_{0}$ is the solar flux at heliocentric distance $r_{0}$ at which the particle number density is $n_{0} ; \Phi(\Theta)$ is the mean volume scattering phase function and the mean total scattering cross-section $\bar{\sigma}$ is defined by

$$
\int_{0}^{\infty} f(s) \sigma(s) \phi(\Theta ; s) d s \equiv \bar{\sigma} \Phi(\Theta) \quad \text { with } \int_{4 \pi} \Phi(\Theta) d \Omega=1 .
$$

The term $Z(\lambda)$ within Eq (1) allows the inclusion of a Gaussian profile with Doppler shift such that

$$
Z(\lambda)=1-\exp \left(-h^{2}\left(\lambda-\lambda_{0}\left[1 \pm V_{r} / c\right]\right)^{2}\right)
$$

the choice of sign being taken according to whether the morning $(+)$ or evening $(-)$ cloud is being considered. For circular orbits, simple geometry shows that

$$
V_{r}=V_{\oplus}\left(\sin ^{\frac{3}{2}} \phi-\sin ^{\frac{3}{2}} \epsilon\right) / \sin ^{\frac{1}{2}} \epsilon
$$

Hong's (1985) photometric model incorporates a scattering phase function given by the weighted combination of three Heyney-Greenstein functions such that

$$
\Phi(\Theta)=\sum_{k=1}^{3} w_{k} \phi_{H G}\left(\Theta ; g_{k}\right) \quad \text { with } \quad\left\{\begin{array}{ll}
w_{1}=0 \cdot 665 & g_{1}=+0 \cdot 70 \\
w_{2}=0.330 & \text { and } g_{2}=-0 \cdot 20 \\
w_{3}=0.005 & g_{3}=-0.81
\end{array}\right. \text {. }
$$

It is obvious from Eq. (1) that inversion of $B_{\lambda}(\epsilon)$ to obtain $V_{r}$ is difficult because of the latter's convolution with $\Phi(\Theta)$. With the above ingredients, the forms of $B_{\lambda}(\epsilon)$ were computed for $\beta$ taken as 12 (see Leinert et al., 1981, although Hong chose $\beta=1$ to avoid a nonphysical dip in the scattering behaviour for $\epsilon<20^{\circ}$ see Hong, 1985, Fig. 5). The exercise was performed on an idealised Gaussian profile with complete absorption at a central wavelength of $4861 \AA$ $(\equiv H \beta)$ rather than taking real lines from a section of the solar spectrum, to allow easier identification of the modifications produced by the model.

\section{Computational Results and Discussion}

As expected, the $R V(\epsilon)$ behaviour was found to be similar to previous models in that for the morning elongation say, the overall profile is red shifted up to $\epsilon \sim 70^{\circ}$ and blue shifted at greater elongations. Two important features emerge, however, these being illustrated in Figs. 1(a) and (b). 
- (A) The profiles display substantial asymmetric broadening and reduction of line depth over the range of small $\epsilon$ up to $70^{\circ}$

- (B) The profile bifurcates at elongations $\sim<30^{\circ}$
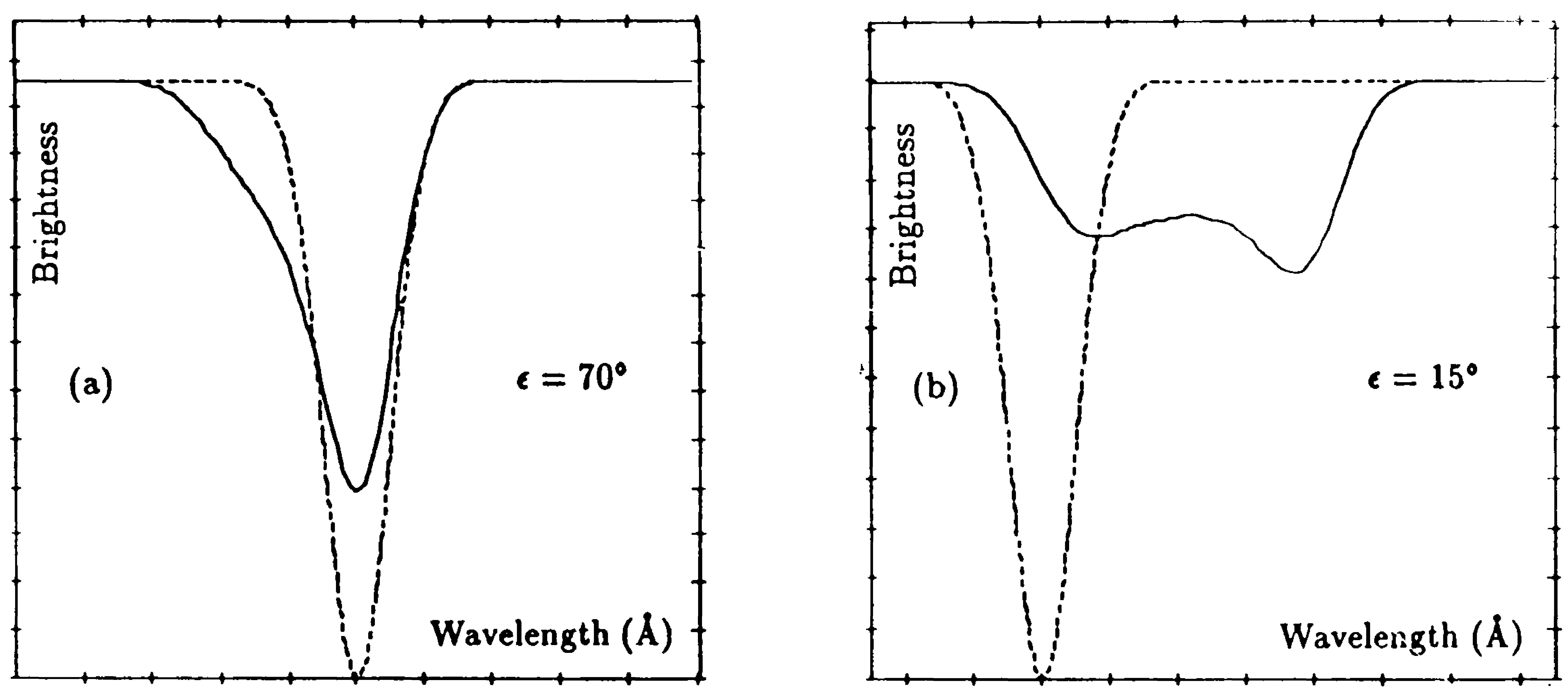

Figure 1. Calculated line profiles for (a) $\epsilon=70^{\circ}$ and (b) $\epsilon=15^{\circ}$ The dashed curves correspond to the original solar Fraunhofer line.

The features noted under (A) are in agreement with previous studies (e.g. see James, 1969) but are more accentuated. Such asymmetries have important consequences for the experimentally obtained $R V(\epsilon)$ curve. For measurements with conventional spectrometers, complete line profiles are recorded; radial velocities may then be determined say from the position of the line minimum. Measurements by a CMRVS instrument, however, correspond more to the line centre of gravity. This effect was appreciated by Fried (1978) but he estimated that such bias away from a line minimum determination was less than $\sim 0.03 \AA$ and can be neglected. With Hong's scattering function, the predicted bias is not trivial; at $\epsilon=40^{\circ}$, for example, the line minimum shift is twice that of the median displacement being $\sim+0 \cdot 2 \AA$ rather than $\sim+0 \cdot 1 \AA$.

It was mentioned earlier that Fried's $R V(\epsilon)$ data have problems of interpretation. This has recently been reconciled by Mukai \& Mann (1993) who applied a model including particles with elliptical orbits. It may be noted that the sense of shift of the $R V(\epsilon)$ curve produced by their model tends to compensate for any bias the CMRVS technique introduces by monitoring asymmetric lines. Matching of models and Fried's $R V$ data resides to some extent in the incorporation of realistic scattering functions such as those proposed by Hong.

The predicted bifurcation noted under (B) is a new feature, with no hint of it in other modelling work. It may be noted that Beavers et al. (1980) used a CMRVS to investigate the $\mathrm{F}$ corona and found multiple dips in their recordings. At the time, two streams of particles in the inner regions of the solar system were proposed in the form of dust in prograde orbits and dust falling into the Sun. Mukai \& Mann (1993) have commented that such separate components cannot be explained by the general dynamic behaviour of circumsolar dust grains. Incorporation of Hong's scattering function in the RV model provides an immediate interpretation for the results of Beavers et al. (1980). 


\section{Conclusion}

The importance of the rôle of particle scattering function with respect to its convolution with dust orbital velocities has been demonstrated for RV studies of the ZL cloud. Hong's phase angle scattering function, successful in mimicking the ZL $B(\epsilon)$ behaviour, has been applied to a model involving dust in circular orbits with the prediction that, in addition to wavelength shifts, ZL profiles will display an $\epsilon$-dependent asymmetry. This has ramifications for the interpretation of RVs obtained by CMRVS instruments and on the evaluation of dust velocities. In addition, at $\epsilon \sim<30^{\circ}$, the zones of dust between the Sun and the Earth and also beyond the Sun with very different velocity vectors both provide important contributions and the predicted profiles display distinct bifurcation, so providing a ready explanation of $\mathrm{RV}$ measurements of the $\mathrm{F}$ Corona by Beavers et al. (1980) without the need to invoke dual dust streams.

Despite the signal-to-noise advantage provided by a CMRVS over a more conventional spectrometer in determining RVs from spectra displaying a simple Doppler shift, it is technically difficult to recover other information on any line distortions. For the future, it would be far better to record the complete line profile, this being attempted in various previous experiments using sequentially scanned Fabry-Pérot interferometers. The time is now right to undertake further observations exploiting the optical advantages of the Fabry-Pérot instrument in association with the 2D/high quantum efficiency of the CCD detector. An extreme technical challenge would be the recording of spectropolarimetric profiles, $p(\lambda)$, so providing more information on the scattering - velocity convolution. It may be noted too, that all experimental arrangements use coelostat mirrors which, because of the ZL polarization accruing along the line of sight, must distort any recorded photometric profile if $p(\lambda)$ also varies across the line.

The paper does not rule out possible asymmetries between the eastern and western elongations and makes no comment on the red shift of the Gegenschein (East \& Reay, 1984), their explanations requiring Doppler shifts associated with some particles having a solar radial component as a consequence of elliptical orbits or as a stream outflow.

\section{References}

Beavers, W.I., Eitter, J.J., Carr, P.H., \& Cook B.C. 1980, ApJ, 238, 349

Blackwell, D.E., \& Ingham M.F. 1961, MNRAS, 122, 129

Clarke, D., Matthews, S.S., Mundell, C.G., \& Weir, A.S. 1995, A\&A, Accepted

East, I.R., \& Reay, N.K. 1984, A\&A, 139, 512

Fried, J W. 1978, A\&A, 68, 259

Hong, S.S. 1985, A\&A, 146, 67

James, J.F. 1969, MNRAS, 142, 45

Leinert, C., Richter, I., Pitz, E., \& Planck B. 1981, A\&A, 103, 177

Mukai, T., \& Mann, I. 1993, A\&A, 271, 530

Ring J.. Clarke, D.. James, J.F., Daehler, M., \& Mack, J.E. 1964, Nature, 202, 167 